\title{
Influence of surface treatment on the structure and properties of proton-exchanged waveguides in lithium niobate
}

\author{
$\underline{\text { S.S. Mushinsky }}^{1}$, I.V. Petukhov ${ }^{1,2}$, V.I. Kichigin ${ }^{1,2}$, O.P. Semenova ${ }^{2}$, A.B. Volyntsev ${ }^{2}$ \\ ${ }^{1}$ PNPPK, 614990, Perm, Russia \\ sergey.mushinsky@gmail.com \\ ${ }^{2}$ Perm State University, 614000, Perm, Russia
}

Lithium niobate (LN) surface condition greatly influences proton exchange (PE) process which in turn influences the structure and properties of PE-waveguides. Influence of several types of surface treatment on the structure and properties of waveguides in congruent LN (X-cut) was investigated in this work.

In particular, it was found that surface treatment in the mixture of sulfuric acid and hydrogen peroxide (Piranha solution) or in the mixture of ammonium hydroxide and hydrogen peroxide (RCA-1) leads to increased value of extraordinary refractive index change at the surface of the crystal $\left(\Delta n_{e}\right)$ after PE and annealing. This may be explained by a better cleaning of the surface of lithium niobate.

On the other hand, surface treatment in oxidizing media containing fluoride ions (mixture of hydrofluoric and nitric acids) leads to decreased values of $\Delta n_{e}$ after PE and annealing. After such treatment, surface composition of LN crystal may change (more specifically, niobium ions depletion and oxygen ions enrichment may occur) which lead to PE inhibition. Since proton concentration in the molten benzoic acid is extremely small, PE happens probably due to hydrogen ions resulting from dissociative adsorption of benzoic acid molecules on the surface of LN.

Congruent LN contains up to $4 \%$ lithium vacancies. Moreover surface layer may contain sufficient number of defect structures formed during wafer manufacturing, e.g. cutting, lapping, polishing. Surface treatment in molten eutectic mixture of $\mathrm{LiNO}_{3} 37.5 \mathrm{~mol} . \%, \mathrm{KNO}_{3} 44.5 \mathrm{~mol} . \%$ and $\mathrm{NaNO}_{3} 18.0$ mol.\% (the mixture was also used for reverse proton exchange (RPE)) could "heal" defects of the surface layer. Nevertheless, LN surface treatment during 1 hour at $330{ }^{\circ} \mathrm{C}$ does not influence subsequent PE. This may be caused by great changes of LN surface during proton exchange process initiated by lithium ion substitution with hydrogen ions. This is why preliminary changes in composition of thin surface layer caused by treatment in lithium-rich melt are rapidly mitigated during proton exchange.

Influence of RPE on waveguide characteristics was studied. RPE rate is greater for unannealed waveguides rather than annealed ones. Structural phase transition rate is greater for RPE than for annealing at the same temperature $\left(330^{\circ} \mathrm{C}\right)$. RPE does not lead to formation of $\kappa_{1}{ }^{-}$ particles which form during post-PE annealing [1].

The research was supported by RFBR (project No. 17-43-590309 p_a).

1. S.S. Mushinsky, V.I. Kichigin, I.V. Petukhov, M.A. Permyakova, D.I. Shevtsov, Ferroelectrics 508, 40 (2017). 